\title{
Zu den Anfängen der Kirchenpolitik Justinians*
}

\author{
Hartmut Leppin
}

Zoras ließ sich nicht alles bieten: Mehrere Jahre hatte der Miaphysit als Stylit in der Region um Amida verbracht, da erschienen - es muß Anfang der dreiBiger Jahre des 6 . Jahrhunderts gewesen $\operatorname{sein}^{1}$ - Anhänger des Konzils von Chalkedon unter seiner Säule und zwangen ihn, von dort herabzusteigen, so berichtet sein Hagiograph, Johannes von Ephesos ${ }^{2}$. Zornentbrannt faßte Zoras daraufhin den Entschluß, zum Kaiser zu gehen, um ihn über den wahren Glauben zu unterrichten; zehn Schüler begleiteten ihn.

Verhindern konnte man Zoras' Marsch auf Konstantinopel offenbar nicht, doch Briefe gingen an die Hauptstadt, um das Eintreffen eines Störenfrieds anzukündigen. Was war zu tun? Sollte man die kleine Asketentruppe einfach zurückschicken? Sollte der Kaiser sie ignorieren? Schließlich hatte Justinian mehr als genug zu tun: Die Verhältnisse an der Ostgrenze erforderten seine ständige Aufmerksamkeit, die Arbeiten an seinen Gesetzessammlungen waren im Gange, im Vandalenreich zeichneten sich vielversprechende Veränderungen ab. Doch der Kaiser verhielt sich ganz anders, als man erwarten würde: Er bereitete den Empfang des Heiligen Mannes gründlich vor,

* Für Hilfe danke ich insbes. M. Keßler (Frankfurt/Main), A. Luther (Berlin) und M. Meier (Tübingen).

1 Vgl. dazu W. H. C. Frend, The Monks and the Survival of the East Roman Empire in the Fifth Century, P\&P 54, 1972, 3-24, hier: 20f. J. A. S. Evans, The Age of Justinian. The Circumstances of Imperial Power, London/New York 1996, 111 bringt die Ankunft des Zoras in Konstantinopel ohne Begründung mit der Ankunft des Severus dort 534/5 in Zusammenhang; S. Ashbrook Harvey, Asceticism and Society in Crisis. John of Ephesus and The Lives of the Eastern Saints, Berkeley - Los Angeles - London 1990, 84 denkt an 535.

2 Hg. und übers. von E. W. Brooks, PO 17-19, Paris 1923-1925. Beat. Or. 2 ist die Vita des Zoras. Ein vergleichbarer Fall ist Berenicianus, s. Ps. Zach. Rhet. 8, 5. Zu Zoras vor allem Ashbrook Harvey, John of Ephesus (wie Anm. 1), $44 \mathrm{f}$. 
denn er verpflichtete Senatoren zum Kommen und versammelte zahlreiche Bischöfe. ${ }^{3}$

Als der Asket vor dem Kaiser erschien, legte der Herrscher ihm, wie ausdrücklich gesagt wird, in versöhnlicher Weise seine theologischen Auffassungen dar. ${ }^{4}$ Was ein rechter syrischer Mönch ist, läßt sich indes von freundlichen kaiserlichen Worten nicht einwickeln. Er kümmert sich auch nicht um die Übrigen, sondern er spricht direkt den Kaiser an. Das tat auch Zoras, in keiner Weise von der Kulisse eingeschüchtert. Er trat also nicht dem Kaiser als einem Repräsentanten von etwas Größerem gegenüber, sondern - in der Manier eines alttestamentarischen Propheten - dem Herrscher als einem einzelnen. ${ }^{5}$ Dabei machte er ihn in grotesk überzogener Weise für die Verfolgung der Kirche Gottes, für das Blutvergießen und für das Konzil von Chalkedon verantwortlich.

Der Kaiser jedoch wagte es nicht, ihn zu schlagen, sondern erklärte mit geballter Faust, man dürfe Chalkedon nicht schmähen, und verlangte von dem Mönch ein Wunder, um seine Behauptungen zu beweisen. Doch Zoras schmetterte ihm entgegen, daß der wahrhaft Gläubige nicht nach Zeichen frage, daß Justinian aber am eigenen Leib ein Zeichen erleben werde. Unter diesen Worten verließ er den Kaiser - offenbar ungehindert. Justinian wurde am nächsten Tag am Haupt getroffen und verlor den Verstand, vor allem aber bildete sich eine entstellende Schwellung am Kopf, so daß Theodora ihn im Palast verbergen mußte. ${ }^{6}$ Erst das Gebet des Zoras vermochte den Kaiser von dem Leid zu erlösen, der zum Dank den Anhängern des Asketen Ver-

3 Ehrenvoller war indes der Empfang des chalkedonischen Sabas, dem Wagen und Bischöfe entgegengesandt wurden, s. Cyr. Scyth. V. Sabae 71.

$4 \mathrm{Da}$ dies versöhnlich geschehen sei, glaubt nicht jeder: Das entsprechende Wort in der

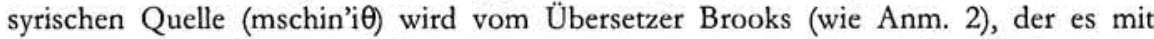
"gently " wiedergibt, angezweifelt: „There is perhaps some error here. If it is right, the intention must be to charge the Chalcedonians with lukewarmness." (23 Anm. 1).

5 Johannes bezieht sich auf 1 . Reg. 22, 31. Das Zitat paßt nach modernen Kriterien nicht ganz, denn es handelt sich um das Gebot des syrischen Königs an den Obersten der Streitwagen, der nur gegen den König Israels, Ahab, kämpfen soll. Wie weit die Szene stilisiert ist, zeigt sich schon daran, daß Zoras für das Griechische eigentlich eines Dolmetschers bedurfte (s. Joh. Eph. Beat. Or. 44 [PO 18, 663]), ein direkter Schlagabtausch zwischen Kaiser und Mönch also nicht möglich war.

6 Möglicherweise wird diese Nachricht von Prokop bestätigt: H. A. 12, 23, wo lediglich von einer gewaltigen Schwellung die Rede ist, aber nicht von Zoras. Ashbrook Harvey, John of Ephesus (wie Anm. 1), 180 Anm. 171 möchte die eingehender erzählte Episode Proc. Aed. 1, 6, 5 darauf beziehen. Doch ist das Krankheitsbild, das in den "Aedificia“ blaß bleibt, in den "Anekdota“ dem näher, was bei Johannes von Ephesos geschildert wird. 
sammlungsfreiheit gewährte. Zoras selbst, der in der nächsten Zeit auf der anderen Seite des Bosporos als religiöse Autorität residierte, blieb im Kontakt mit dem Kaiser. ${ }^{7}$

Eine Anekdote wie die eben referierte Auseinandersetzung zwischen Kaiser und Heiligem Mann zeigt Justinian in einer vermeintlich ungewohnten Rolle. ${ }^{8}$ Der Kaiser, der im neuzeitlichen Geschichtsbewußtsein wie kein anderer den Machtanspruch des spätantiken Kaisertums verkörpert, nimmt es hin, daß ein schwacher Mönch ihn tadelt - ein Mönch, der sich überdies seinen dogmatischen Vorstellungen verweigert. Gerade dies scheint mir aber typisch für die Kirchenpolitik nicht nur in den frühen Jahren Justinians zu sein, zu der ich einige Überlegungen anstellen möchte. Justinians Position war, so wird im allgemeinen behauptet, zu Beginn seiner Regierung, wie es sein Onkel vorgegeben habe, von einer energischen Verfolgungspolitik bestimmt, die in den dreißiger Jahren kurzzeitigen Aussöhnungsversuchen mit den Miaphysiten gewichen sei, doch um 536 habe der Kaiser wieder eine hart chalkedonische Linie verfolgt. ${ }^{9}$ Mit einem von Eduard Schwartz eingeführten Bild spricht man gerne von einem Zickzackkurs ${ }^{10}$.

7 Beat. Or. 2, PO 17, 25 f. Daß ein Mönch einer anderen Glaubensrichtung vom Kaiser mit Freundlichkeit empfangen werden konnte, zeigt auch die Begegnung zwischen Anastasius und dem Chalkedonier Sabas, s. Cyr. Scyth. V. Sab. 51, wo der Kaiser als

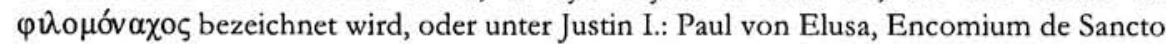
Theognio 21, hg. von S. Vailhé, AB 10, 1891, 78-113.

8 Vgl. aber zur Zugänglichkeit des Kaisers Proc. H. A. 13, 1-3; 15, 12.

9 Grundlegend nach wie vor E. Stein, Histoire du Bas-Empire, Paris/Brüssel 1949, Bd. 2, 369ff.; vgl. ferner R. Haacke, Die kaiserliche Politik in den Auseinandersetzungen um Chalkedon (451-553), in: A. Grillmeier/H. Bacht (Hgg.), Das Konzil von Chalkedon. Geschichte und Gegenwart, Bd. 2: Entscheidung um Chalkedon, Würzburg 31974, 95177, hier: 152 ff.; J. Meyendorff, Imperial Unity and Christian Divisions, Crestwood 1989, $207 \mathrm{ff}$; immer noch nützlich H. S. Alivisatos, Die kirchliche Gesetzgebung des Kaisers Justinian I. (Neue Studien zur Geschichte der Theologie und der Kirche 17), Berlin 1913; W. H. C. Frend, The Rise of the Monophysite Movement. Chapters in the History of the Church in the Fifth and Sixth Centuries, Cambridge 1972, 255ff.; vgl. O. Mazal, Justinian I. und seine Zeit. Geschichte und Kultur des Byzantinischen Reiches im 6. Jahrhundert, Köln u. a. 2001, $203 \mathrm{ff}$.

10 E. Schwartz, Zur Kirchenpolitik Justinians, SBAW 1940, Nr. 2, 32-81, hier: 32; auch in: ders., Gesammelte Schriften, Bd. 4, Berlin 1960, 276-326, hier: 276; grundlegend auch ders., Kyrillos von Skythopolis (Texte und Untersuchungen 49, 2), Leipzig 1939; dort ist 395 von der „unsicher schwankenden Passivität des Kaisers“ die Rede. S. zum Bild des Zickzackkurses die Belegsammlung bei M. Meier, Das andere Zeitalter Justinians. Kontingenzerfahrung und Kontingenzbewältigung im 6. Jahrhundert $\mathrm{n}$. Chr. (Hypomnemata. Untersuchungen zur Antike und zu ihrem Nachleben 147), Göttingen 2003, 274 Anm. 215 , der sich seinerseits davon distanziert. 
Einen anderen Ansatz hat jüngst indes Karl-Heinz Uthemann verfolgt, indem er in überzeugender Weise die Kohärenz der theologischen Position Justinians während der ersten Jahre herausgearbeitet hat. ${ }^{11}$ In diese Richtung möchte ich weiterdenken, ${ }^{12}$ indem ich aufzuzeigen versuche, daß die Politik Justinians während der ersten Jahren durchaus konsistent war, ${ }^{13}$ und zwar insofern, als er zum einen nach einer gemeinsamen Linie von Miaphysiten und Chalkedoniern suchte, zum anderen den Habitus eines demütigen Herrschers einnahm, der sich von Glaubensautoritäten belehren lassen wollte; man könnte in diesem Sinne von einer reaktiven Grundhaltung sprechen.

Noch als Caesar formulierte Justinian in einem Brief an den Papst Hormisdas eine theopaschistische Lehre, ${ }^{14}$ also die Lehre, daß einer aus der Trinität gelitten habe. Diese Lehre wird mit den Aktivitäten skythischer Mönche in Verbindung gebracht, die sich um eine anti-nestorianische Interpretation der Beschlüsse von Chalkedon bemühten und insofern den Miaphysiten entgegenkamen; indes wurden die Lehren dieser Mönche vom Papst abgelehnt. Bereits hier zeigt sich somit Justinians Neigung zum Ausgleich mit den Mi-

11 K. H. Uthemann, Kaiser Justinian als Kirchenpolitiker und Theologe, Augustinianum 39, 1999, 5-83, hier: 46f. Der Aufsatz bietet eine sorgsam aufbereitete Darstellung der Stationen von Justinians Kirchenpolitik mit ausführlichen Literaturhinweisen; weniger nuanciert ist P. T. Gray, The Defense of Chalcedon in the East (451 - 553), Leiden u. a. 1979, 53ff.; seine Position ist zusammengefaßt in „The Legacy of Chalcedon. Christological Problems and their Significance", in: M. Maas ( $\mathrm{Hg}$.), The Cambridge Companion to the Age of Justinian, Cambridge 2005, 215-238. Gray schlägt vor, von Anti-Chalkedoniern zu sprechen; das aber würde auch Dyophysiten bzw. Nestorianer ei nschließen.

12 In manchem ergeben sich Berührungspunkte zu K. L. Noethlichs, Iustinianus (Kaiser), RAC 19, 1999, 668-763, hier: 689. Meyendorff, Imperial Unity (wie Anm. 9), insbes. 246, wendet sich zwar auch gegen das Bild vom „Zickzack“, indem er die konsequent chalkedonische Orientierung Justinians herauszuheben versucht, beurteilt aber sein Verhältnis zu den Miaphysiten ganz anders, als es hier getan wird.

13 Üblicherweise sieht man 531 einen Bruch oder jedenfalls Wandel, s. z. B. Schwartz, Kirchenpolitik Justinians (wie Anm. 10), 39-284; J. Speigl, Die Synode von 536 in Konstantinopel, OS 43, 1994, 105-153, hier: 108f., der 108 von einer „Wiedervereinigungspolitik seit 531“ spricht. Meier, Zeitalter Justinians (wie Anm. 10), 278, der in den 30er Jahren lediglich eine „kurzfristige Gesprächsbereitschaft" erkennt oder 288 eine dauerhaft antimonophysitische Politik erkennt.

$14 \mathrm{Zu}$ ihnen A. Grillmeier, Jesus der Christus im Glauben der Kirche, Bd. II 2: Die Kirchen in Konstantinopel im 6. Jahrhundert, Freiburg u. a. 22004, 338ff.; J. Speigl, Formula Iustiniani. Kircheneinigung mit kaiserlichen Glaubensbekenntnissen (Codex Iustinianus I 1, 5-8), OS 44, 1995, 105-134, hier: 106ff. Meier, Zeitalter Justinians (wie Anm. 10), $215 \mathrm{ff}$ betrachtet die Formel als ungeeignet für eine Kompromißfindung, urteilt aber dabei möglicherweise zu sehr ex eventu, da sie in der Tat die Einheit nicht herbeiführte. Doch das war in den ersten Regierungsjahren nicht absehbar. 
aphysiten. Vasiliev erkennt sogar einen mildernden Einfluß Justinians auf die Politik Justins, sieht aber in einem Häretikergesetz, das offenbar 527 während der gemeinsamen Regierungszeit Justins und Justinians entstand, einen Ausdruck der Verschärfung. Doch fällt bei diesem Gesetz auf, daß es die Miaphysiten nicht ausdrücklich nennt. ${ }^{15}$

Und das scheint ein spezifisch justinianisches Element zu sein: Bestimmungen zu religiösen Fragen ergehen während der ersten Regierungsjahre Justinians in dichter Folge. ${ }^{16}$ Als er 527 die Alleinherrschaft antrat, tat er etwas, was an Theodosius den Großen gemahnte: Er veröffentlichte bald ein Glaubensbekenn tnis. ${ }^{17}$ Zwar klingt darin auch die theopaschistische Formel an, ${ }^{18}$ doch nicht in der klassischen, im Streit gewissermaßen verbrauchten Version. Auch sonst sind die Formulierungen des Textes dergestalt, daß eine eindeutige Festlegung auf bestimmte, besonders umstrittene Schlagworte vermieden wird; vor allem das Wort $\varphi v ́ \sigma 1 \zeta$, das in der Debatte so wichtig war, wird vermieden. ${ }^{19}$ Als essentiell definiert Justinian in der Praefatio seines Gesetzes gegen die Häretiker das Homoousios und den Glauben, daß Maria Theotokos, die Gottesgebärerin sei, worin ein eindeutig anti-nestorianisches Bekenntnis liegt. Anders als Theodosius benennt Justinian auch keine Normalbischöfe, andererseits aber explizit bestimmte häretische Gruppen bzw.

15 CJ 1, 5, 12; 1, 5, 12, 17 trifft eine Sonderregelung für arianische Goten. A. A. Vasiliev, Justin the First. An Introduction to the Epoch of Justinian the Great (DOP Studies 1), Cambridge/Mass. 1950, 132ff, insbes. $221 \mathrm{ff}$.

16 Vgl. M. Maas, Roman History and Christian Ideology in Justinianic Reform Legislation, DOP 40, 1986, 17-31, hier: 25; Speigl, OS 43, 1994 (wie Anm. 13), 111ff.; Meier, Zeitalter Justinians (wie Anm. 10), 200.

17 CJ 1, 1, 5; die Formulierungen sind weitaus vager als im Henotikon Zenos (Evagr. H. E. 3, 14; anders Haacke, Chalkedon [wie Anm. 9], 154). Die Datierung - gewöhnlich wird 527 angegeben - ist nicht ganz gesichert; als Teil des „Codex Iustinianus“ muß der Text aus den Anfängen der Regierung stammen; CJ 1, 1, 6, 3 verweist offenbar darauf zurück. Ohne Begründung datiert Mazal, Justinian (wie Anm. 9), 205 den Text auf 533 und meint daher, daß Justinian zu Beginn seiner Regierungszeit anti-miaphysitisch gesonnen gewesen sei. Zu der theologischen Offenheit vgl. Noethlichs, Iustinianus (wie Anm. 12), 689, der die Nähe zu den Debatten des Konzils von Ephesos hervorhebt; zu Theodosius I. vgl. H. Leppin, Theodosius der Große - Auf dem Weg zum christlichen Imperium, Darmstadt 2003, 68ff.

$18 \int 1$. Anders akzentuiert bei Grillmeier, Jesus der Christus (wie Anm. 14), 355. Möglicherweise ist auf diese Passage die Äußerung Vict. Tun. s. a. 529 (MGH AA XI, 197)zu beziehen, der seiner Grundhaltung gemäß hier Theodora am Werke und Verfolgungsmaßnahmen eingeleitet sieht.

19 Das muß auch Speigl, OS 43, 1994 (wie Anm. 13), 113 anerkennen, obwohl er bemüht ist, dem Text ein klares Bekenntnis Justinians zu entlocken. 
ihre Anführer. Darunter findet sich zwar mit Eutyches ( $(3)$ jemand, den Chalkedonier gerne den Miaphysiten zuschlugen - aber dieser Lehrer wurde gewöhnlich auch von den Miaphysiten abgelehnt. Anders als in einem späterem, 541 vorgelegten Häretikerkatalog, ${ }^{20}$ der die Anhänger des Severus anführt, werden keine Miaphysiten ausdrücklich genannt. Damit war, und darin setze ich mich von der üblichen Interpretation ab, die scharf antimiaphysitische Politik Justins ${ }^{21}$ zwar nicht formell zurückgenommen, faktisch aber aufgehoben. Justinian signalisierte wohl, ohne seinen Onkel zu desavouieren, ein Ende der harten Repressionspolitik gegenüber den Miaphysiten.

Dieser Öffnung gegenüber den Miaphysiten korrespondierte eine Verschärfung der Politik gegenüber den eigentlichen Häretikern im Sinne Justinians, aber auch Manichäern - gegen die er besonders streng vorging -, Juden und Samaritanern, die er zunehmend unter diesem Begriff faßte. Ihnen wurden wesentliche bürgerliche Rechte genommen und die Religionsausübung untersagt. ${ }^{22}$ Was in diesen Edikten angelegt ist, prägte die Politik Justinians für die nächsten Jahre: Dogmatische Äußerungen, sofern er sie überhaupt machte, blieben vage, dafür wurden gemeinsame Gegner von Chalkedoniern und Miaphysiten mit ungewöhnlicher Energie bekämpft. Heiden gerieten vor allem 529/30 unter Druck, wobei es Justinian inzwischen nicht mehr allein um die Praktiken des Heidentums, sondern um die Gesinnung der Menschen ging. ${ }^{23}$ Die bekannteste Folge der kaiserlichen Politik bestand darin, daß die Akademie in Athen allmählich ein Ende fand und die Philosophen zunächst in das Persische Reich zogen. ${ }^{24}$ Die Samaritaner wurden ebenfalls hart bedrängt - nicht zuletzt aufgrund der Anklagen eines Mönches; ${ }^{25}$ diese Politik mündete in einen verheerenden Aufstand. Auf der anderen Seite erschienen

20 Nov. Iust. 109 pr.; vgl. 115, 3, 14 (542), wo ausdrücklich von den Akephalen, eine verbreitete Bezeichnung für die Miaphysiten, die Rede ist, und zwar in Ergänzung von CJ 1, 5,19 von 529.

21 Vgl. Stein, Histoire II (wie Anm. 9), 230ff.; Haacke, Chalkedon (wie Anm. 9), $141 \mathrm{ff.}$

22 CJ 1, 5, 12 (dafür, daß es noch in der Zeit der gemeinsamen Herrschaft erlassen worden ist, spricht CJ 1, 5, 18,4); auch die anderen antihäretischen Bestimmungen haben zunächst keinen spezifisch anti-miaphysitischen Akzent, s. CJ 1, 5, 13-22; Nov. Iust. 11, 5; 17,$11 ; 37,5-1$, s. den Überblick bei Alivisatos, Justinian (wie Anm. 9), $32 \mathrm{ff}$.

23 Vgl. dazu Meier, Zeitalter Justinians (wie Anm. 10), 203ff., der zu Recht betont, daß mit diesen Verfolgungen auch politische Zwecke erfüllt werden sollten; vgl. Theoph. a. m. 6022; Mal. 18, 42 Thurn; Proc. H. A. 11, 31 f.

24 Vgl. dazu Al. Cameron, The Last Days of the Academy at Athens, PCPhS n. s. 195, 1969, 7-29; R. Thiel, Simplikios und das Ende der neuplatonischen Schule in Athen (AAWM 1999, Nr. 8), Stuttgart 1999; Meier, Zeitalter Justinians (wie Anm. 10), $207 \mathrm{ff}$.

25 Cyr. Scyth. V. Sab. 71. 
528 zwei Barbarenfürsten in Konstantinopel, die sich und damit ihr Volk zum Christentum bekehren ließen. ${ }^{26}$

Der Kaiser war somit offenkundig bestrebt, die Erwartung, er müsse ein Vorkämpfer des Glaubens sein, zu erfüllen. Darin eiferte er dem Vorbild seines Onkels nach, doch mit einem erkennbar moderateren Kurs gegenüber den Miaphysiten, die er, wenn ich recht habe, nicht mehr als Häretiker bezeichnet sehen wollte.

Für 531 hört man zwar davon, daß die Miaphysiten von Antiocheia und überhaupt im Osten sich bedrängt gefühlt hätten, doch ging dies auf die Initiative eines - in seiner Region durchaus mächtigen - Bischofs zurück, des entschieden anti-miaphysitischen Ephraim. ${ }^{27}$ Unter den Verhältnissen des 6. Jh.s ist nicht zu erwarten, daß die kaiserliche Religionspolitik in allen Reichsteilen unmittelbar wirksam wurde; formell aufgehoben waren die Regelungen Justins ja nicht, und das Häretikergesetz Justinians erlaubt vielfältige Deutungen, so daß Ephraim sich ganz im Recht gefühlt haben kann.

Jedenfalls steuerte Justinian ohne Zweifel in Konstantinopel weiter einen anderen Kurs: Der miaphysitische Historiker Ps.-Zacharias Rhetor berichtet, daß der Kaiser im fünften Jahr seiner Regierung Miaphysiten aus der Verbannung zurückgerufen, somit eine Maßnahme seines Vorgängers rückgängig gemacht habe. ${ }^{28}$ Dies lag in der Konsequenz der bisherigen Politik Justinians und steht in einem engen zeitlichen, bei Zacharias sogar in einem darstellerischen Zusammenhang mit dem Nika-Aufstand. Ob auch ein kausaler $\mathrm{Zu}$ sammenhang bestand, muß offen bleiben. Immerhin war seit diesem Ereignis der Faktor städtische Bevölkerung, die zu einem großen Teil miaphysitische Tendenzen handgreiflich bekämpft hatte, besser unter Kontrolle - insofern hatte der kaiserliche Handlungsspielraum sich erweitert.

26 Der Heruler Grepes bzw. Gretes (PLRE IIIA, 555) sowie der Hunne Grod (PLRE IIIA, 557 f.); vgl. zum komplexen Zusammenhang zwischen äußerer Politik und Religionspolitik K. L. Noethlichs, Quid possit antiquitas nostris legibus abrogare? Politische Propaganda und praktische Politik bei Justinian I. im Lichte der kaiserzeitlichen Gesetzgebung und der antiken Historiographie, ZAC 4, 2000, 116-132, hier: 126.

27 Mal. 18, 64 Thurn; bei Joh. Eph. Beat. Or. werden verschiedentlich (21 [PO 17, 293f.]; 35 [PO 18, 620f.]; 58 [PO 19, 224]; vgl. 24 [PO 18, 522f.]; 50 [PO 19, 153]) ausdrücklich Kirchenleute, zumal Ephraim von Antiocheia, nicht aber Justinian verantwortlich gemacht. Das läßt sich nicht allein aus der prinzipiell loyalen Haltung des Autors gegenüber dem Kaisertum erklären, da er bisweilen - etwa im Kontext der Vita des Zoras - auch Kritik am Kaiser übt.

28 Zach. Rhet. H. E. 8, 5; vgl. 8, 4; 9, 15; vgl. Joh. Eph. Beat. Or. 35 (PO 18, 619 f.), der von einer Erlaubnis spricht, in das alte Kloster zurückzukehren. 
Möglicherweise gehört der Marsch des Zoras nach Konstantinopel und sein Aufenthalt dort in jenen Kontext, auch wenn Johannes von Ephesos etwas anderes glauben machen will. ${ }^{29}$ Nicht alle Miaphysiten hatten indes bereits so viel Vertrauen zum Kaiser und dem unruhigen Mob von Konstantinopel gefaßt, daß sie sich tatsächlich in die Hauptstadt begaben; Severus von Antiocheia, ihr Exponent, etwa blieb zunächst fern. Immerhin stand denen, die kamen, jetzt ein verläßlicher Treffpunkt zur Verfügung, der kaiserliche Palast, in dem Theodora über die Sicherheit ihrer Glaubensfreunde wachte, ${ }^{30}$ auch später noch, als sie ungleich stärker bedrängt wurden.

Einen weiteren Versuch eines Ausgleichs zwischen den theologischen Richtungen bedeutet es, daß Justinian einige der Miaphysiten, abgesetzte Bischofe aus dem Antiochener Patriarchat sowie ausgewählte Chalkedonier für 532 zu einem ungefähr paritätisch besetzten Religionsgespräch nach Konstantinopel einlud. ${ }^{31}$ Die Quellenlage für dieses Ereignis ist einzigartig: Wir haben einen chalkedonisch orientierten Bericht des Innocentius von Maroneia, eines der Teilnehmer, in Briefform ${ }^{32}$ sowie einen miaphysitisch ausgerichteten eines unbekannten Autors, den Sebastian Brock vor einiger Zeit entdeckt hat und der durch einen zweiten, kürzeren, der schon länger bekannt war, ergänzt wird. ${ }^{33}$

29 Vgl. etwa Schwartz, Kyrillos (wie Anm. 10), 392f.; Speigl, OS 43, 1994 (wie Anm. 13), 109.

30 Eine plastische Schilderung bei Joh. Eph. Beat. Or., 47 (PO 18, 676-684); vgl. Ashbrook Harvey, John of Ephesus (wie Anm. 1), 86ff. Ihren Einfluß sollte man indes keinesfalls überschätzen, zumal sie das eindeutige Bekenntnis zu Chalkedon nicht verhindern konnte, s. H. Leppin, Theodora und Iustinian, in: H. Temporini-Vitzthum ( $\mathrm{Hg}$.), Die Kaiserinnen Roms. Von Livia bis Theodora, München 2002, 437-481; H. Leppin, Kaiserliche Kohabitation: Von der Normalität Theodoras, in: C. Kunst/U. Riemer (Hgg.), Grenzen der Macht. Zur Rolle der römischen Kaiserfrauen, Stuttgart 2000, 75-85.

31 S. P. Brock, The Orthodox-Oriental Conversation of 532, Apostolos Barnabas 41, 1980, 219-228; auch in: ders., Syriac Perspectives on Late Antiquity, Aldershot u. a. 1984, Nr. XI; J. Speigl, Das Religionsgespräch mit den severianischen Bischöfen in Konstantinopel im Jahre 532, AHC 16, 1984, 264-285; Grillmeier, Jesus der Christus (wie Anm. 14), 244ff.; $361 \mathrm{ff}$; Uthemann, Augustinianum 39, 1999 (wie Anm. 11), 27ff. Zum Datum Stein, Histoire II (wie Anm. 9), 378. - Frend, Monophysite Movement (wie Anm. 9), 260ff. vermutet, daß der Wunsch der Miaphysiten, eine Kirchenorganisation durch eigene Weihungen $u$. ä. aufzubauen, der Anlaß für Justinians Maßnahme war.

32 E. Schwartz (Hg.), Acta conciliorum oecumenicorum, 4 Bde., Berlin - Leipzig 1914 1984, im folgenden zitiert als ACO mit Angabe des Bandes und des Faszikels, hier: ACO IV 2, 169-184. Es handelt sich um die lateinische Übersetzung eines griechischen Briefes.

33 S. Brock, The Conversations with the Syrian Orthodox under Justinian, OCP 47, 1981, 87-121; auch in: ders., Studies in Syriac Christianity. History, Literature and Christianity, 
Aus den Gemeinsamkeiten der Texte läßt sich der Verlauf der Verhandlungen im Groben rekonstruieren. ${ }^{34}$ Die Debatten, denen Gespräche des Kaisers mit beiden Gruppen vorausgingen, erstreckten sich über drei Tage. Sie wurden nicht vom Kaiser, der dies ausdrücklich ablehnte (I, $\ 5$ ), geleitet, ${ }^{35}$ sondern von Strategius, einem Angehörigen des höchst einflußreichen Geschlechts der Apionen, der den magister officiorum vertrat und es später zum comes sacrarum largitionum bringen sollte. ${ }^{36}$ Am dritten Tag nahm der Kaiser, der sich an den Abenden immer hatte über den Stand der Gespräche informieren lassen, ${ }^{37}$ das Gespräch auf, in der Hoffnung, eine kompromißbereite Gruppe vorzufinden, doch blieb die Distanz zwischen den Diskutanten erhalten.

Es versteht sich, daß die Verfasser der jeweiligen Texte darüber unterschiedlicher Ansicht sind, welche der beiden Gruppen die stärkeren Argumente vorgetragen habe. Ebenso wenig überrascht es, wenn nur Innocentius zu berichten weiß, daß mit Philoxenus von Doliche sich ein Miaphysit bei diesem Anlaß zum Chalkedonismus bekehrt habe. Wichtig sind für meine Fragestellung die Unterschiede in der Darstellung der Rolle des Strategius und des Kaisers.

Innocentius, der Chalkedonier, berichtet über eine an die Orientales, die Miaphysiten, gerichtete Einleitungsrede des Strategius. Darin heißt es, der Kaiser habe das Gespräch einberufen non ex auctoritate principali, sed paterna et sacerdotali compunctione (I, $\mathbb{8}$ ), nicht aufgrund seiner kaiserlichen Autorität, sondern aufgrund seiner väterlichen und priesterlichen Gewissensnot. Der Kaiser will also hier nicht als Herrscher in Erscheinung treten. Allerdings ist sein Anspruch, aus väterlicher und priesterlicher Gewissensnot - gemeint ist natürlich die Gewissensnot über die Lage der Kirche - ein Religionsgespräch einzuberufen, in gewisser Weise noch höher, da der Kaiser hier nicht zwischen der weltlichen und der kirchlichen Sphäre trennt, sondern sich mit den

Aldershot u. a. 1992, Nr. XIII) sowie PO 13, 192-196, der geraffte Text, bei Brock a.a.O. 113-117. Mit Brock be nenne ich die längere Version als $\mathrm{H}$, die kürzere als $\mathrm{S}$, den Brief des Innocentius als I.

34 Vgl. die Übersicht bei Brock, OCP 47, 1981 (wie Anm. 33), 118 f.

35 Nicht einmal dem Wunsch der Miaphysiten, ihr Glaubensbekenntnis vor ihm und hohen Würdenträgern verlesen zu lassen, kommt er nach, sondern erklärt, er werde es lesen, wenn er die Zeit dafür finde $(\mathrm{H}, \mathbb{\$} 1)$ - allerdings sorgt er dafür, daß die Chalkedonier es

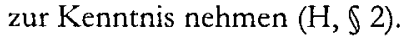

36 PLRE II, 1034-1036.

37 Nach Auskunft die Miaphysiten taten dies allerdings allein die Chalkedonier, s. H, \& 33 f. 
Priestern in eins setzt, ${ }^{38}$ wenn er sich mit dem Adjektiv paternus nicht sogar über sie stellt. Strategius selbst berichtet den Gesprächsteilnehmern von der Bekehrung seines eigenen Vaters zum chalked onischen Glauben - Chalkedon wird ausdrücklich erwähnt - durch die Kaiser ${ }^{39}$ und setzt damit einen deutlichen Akzent, auch wenn er die Beteiligten zu einem friedlichen Gespräch auffordert, ${ }^{40}$ wie es der Kaiser schon im Vorgespräch gegenüber denn Chalkedoniern getan hatte $(\mathrm{I}, \S 4 \mathrm{f}$ ) $)$.

In den miaphysitischen Quellen bleibt diese Rede unerwähnt; bei ihnen erscheint Strategius indessen als jemand, der verschiedentlich in die Debatten gezogen wird, während er zufolge des Eindrucks, den Innocentius vermittelt, nach seiner Einführungsrede gar nicht mehr eingreift. So lehnt er den Wunsch der Miaphysiten ab, ein Protokoll führen zu lassen, mit der Begründung, er sei dazu nicht befugt $(H, \$ 11)$. An anderer Stelle kommt Strategius den Miaphysiten entgegen, indem er ihnen die Verlesung eines für die dogmatischen Debatten wichtigen Briefes gestattet $(\mathrm{H}, \mathbb{1} 19)$.

Ganz verschieden wird der dritte Tag geschildert. Laut Innocentius sucht der Kaiser zunächst das Gespräch mit dem Bischof von Konstantinopel, Epiphanios, und mit den Chalkedoniern, wobei - wie anläßlich der Auseinandersetzung mit Zoras - der Senat zugegen ist, an dessen Seite die chalkedonischen Bischöfe sich niederlassen dürfen. Auch jetzt geht es offenbar darum zu zeigen, daß der Kaiser nicht für sich allein stehe, sondern etwas Größeres repräsentiere. Danach lädt Justinian die übrigen Teilnehmer hinzu, und spricht mit ihnen, wie Innocentius beteuert, bemerkenswert mild $(I, \S 80)$ - obwohl die Sitzverteilung ja schon ein eindeutiges Signal der Zurücksetzung ist. Einmal mehr versucht Justinian, seine theopaschistischen Vorstellungen ins Spiel zu bringen, denen die Chalkedonier unter ausführlichen Qualifizierungen zustimmen (I, $₫ 83-86$ ).

Nach Abschluß dieser Gespräche lädt der Kaiser die Chalkedonier erneut in den Palast, um dort zu berichten, daß er im Michaels-Oratorium ${ }^{41}$ für eine Einigung der Kirche gebetet und so Philoxenus gewonnen habe (I, \87-89).

38 Papst Vigilius erkennt allerdings Justinian auch noch einen sacerdotalis animus zu (Coll. Av. 92,2 ), und dieses Zeugnis ist nicht isoliert, so daß man jener Äußerung kein zu großes Gewicht beimessen sollte.

$39 \mathrm{Zu}$ Apion (2), der sich unter Anastasius als Miaphysit hervorgetan hatte, PLRE II, $111 \mathrm{f}$. Die Hintergründe der Konversion sind nicht klar.

40 Wobei das Gespräch von vornherein nicht ganz symmetrisch ist, weil die Miaphysiten lediglich Fragen an die Chalkedonier stellen sollen.

41 Zu seiner mutmaßlichen Lage im Bereich des Palastes s. R. Janin, La géographie ecclésiastique de l'empire byzantin, Bd. I 3, Paris 1953, 355. 
Fortan habe der Kaiser, so schließt Innocentius, durch seine Güte noch viele Miaphysiten, wenn auch keine Bischöfe bekehrt. Das chalkedonische Schreiben endet also mit der Verheißung, daß das Religionsgespräch Grundlagen für ihre weitere Stärkung geschaffen habe.

Während in der chalkedonischen Version der Abschlußtag zu einer Feier kaiserlicher Milde wird und der Kaiser als weitgehend zurückhaltend in dogmatischen Fragen erscheint, zeigen die Miaphysiten, die den dritten Tag erheblich ausführlicher, allerdings nicht ohne Unterschiede schildern, Justinian stärker in die Auseinandersetzungen verstrickt. Ihre Berichte atmen das Gefühl der Benachteiligung, andererseits zeichnen sie ein lebendiges Bild vom Kaiser bei seinen Bemühungen, einen Kompromiß zu finden. Der Kaiser versucht, eine Brücke zu bauen, indem er erklärt, daß die Miaphysiten eigentlich rechtgläubig seien und sich lediglich in der Abendmahlsfrage störrisch anstellten $(H, \ 36)$. Die Miaphysiten antworten ausweichend. Danach fordert der Kaiser die Miaphysiten auf, sich mit den Bischöfen von Rom, Alexandreia, Antiocheia und Jerusalem in Verbindung zu setzen, um deren Posi-

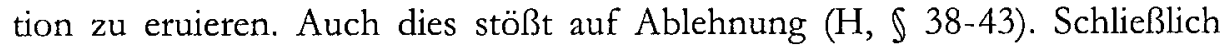
fragt der Kaiser enttäuscht, welches Mittel die Bischöfe denn vorschlagen würden, um einen Kompromiß zwischen den vier Patriarchaten zu erreichen, und erhält keine Antwort ( $\mathrm{H}, \S 40 \mathrm{f}$.). Als der Kaiser das Verhältnis der Miaphysiten zum Patriarchen von Alexandreia zu eruieren versucht, bricht der Text $a b$.

Der geraffte miaphysitische Text berichtet wie $\mathrm{H}$, daß der Kaiser die Miaphysiten dazu zu bringen sucht, sich mit den vier Patriarchaten in Verbindung zu setzen $(S, \mathbb{S}$ ), ebenso wird erwähnt, daß der Kaiser um einen Vorschlag bittet, den Frieden in den Kirchen zu erreichen $\left(S, \int 5\right)$. Ansonsten bringt die kürzere Fassung - die möglicherweise dem verlorenen Teil des detaillierten Berichtes entspricht - ganz andere Aspekte. So versucht der Kaiser hier, den Bischöfen die Zusage abzugewinnen, daß sie keine Weihen oder Taufen außerhalb ihres Kreises vornehmen würden; doch diesem Ansinnen entziehen sie sich. ${ }^{42}$ Justinian macht noch einen sehr weitgehenden Kompromißvorschlag, nämlich das Konzil von Chalkedon insofern zu akzeptieren, als es Eutyches verurteilt habe ${ }^{43}$ - womit er zwar im Geist seiner bereits referierten Gesetzgebung weiterdenkt, doch mit dem großen Unterschied, daß jetzt der Begriff „Chalkedon“ auftaucht. Wieder verweigern sich die Bischöfe. Als der Herrscher auf eine persönliche Unterredung mit ihrem

42 S, $₫ 4$; vgl. zum Thema $H, \S 10$.

$43 \mathrm{~S}, \mathbb{9}$. Damit verzichtete er letztlich auf die theologischen Inhalte der Konzilsbeschlüsse, vgl. Grillmeier, Jesus der Christus (wie Anm. 14), 438. 
Oberhaupt Severus drängt, behaupten die Bischöfe, sie würden seinen Aufenthaltsort nicht kennen ( $\left.S, \int 4\right)$. Zwar wird der Ton zeitweise gereizter; der Kaiser droht mit Verbannung, doch geht das Motiv in der weiteren Debatte unter. Wie die Diskutanten auseinandergehen, wird nicht mehr berichtet.

Gemeinsam ist beiden miaphysitischen Texten, daß die Miaphysiten sich auf Argumentationen nicht einlassen, sondern den kaiserlichen Wünschen mit formalen Einwänden - sie wüßten bestimmte Dinge nicht, sie könnten anderes nicht entscheiden etc. - entgegentreten. Zwar wird deutlich, daß die Miaphysiten sich benachteiligt fühlen, doch ein hartnäckiges Bemühen des Kaisers um einen Kompromiß wird ebenfalls sichtbar.

Es ist schwer zu entscheiden, welcher Quelle im Einzelnen zu folgen ist ich neige insgesamt mehr den allen Anschein nach weniger stilisierten, die eigenen Vertreter nicht immer in ein vorteilhaftes Licht rückenden miaphysitischen Texten zu, die trotz ihres polemischen Untertons ein stimmiges Bild des kaiserlichen Verhaltens bieten -, doch ist eines klar, und das sei noch einmal betont: Der Kaiser ist selbst in der miaphysitischen Version, die ihn natürlich als Freund der Chalkedonier zeichnet, nicht das dogmatische Rauhbein, als welches er später erscheint. Er ist vielmehr jemand, der es zu vermeiden sucht, in die inhaltlichen Debatten hineingezogen zu werden. Justinian gerät auch nicht in Erregung über das Verhalten der Miaphysiten, das durch Starrheit und Ausweichmanöver gekennzeichnet ist. Er verzichtet darauf, die kaiserliche Macht auszuspielen; die typische Untugend des Herrschers, die Neigung zum Zorn, bricht nicht durch. Offenbar gebärdete Justinian sich, diesen Schluß erlauben beide Versionen, nach wie vor als der Kaiser, der bereit war, alle anzuhören.

Allerdings nahm er anscheinend für sich in Anspruch, in einer Nahbeziehung zu Gott zu stehen, wie sein Gebet im Michaels-Oratorium verdeutlicht und letztlich auch die Motivation der Einladung zum Gespräch. Vergleicht man den Verlauf dieses Religionsgesprächs mit dem eines anderen, nämlich jenem von Konstantinopel 383, zu dem Theodosius der Große geladen hatte, ${ }^{44}$ so werden bemerkenswerte Unterschiede deutlich.

Theodosius hatte laut dem Kirchenhistoriker Sokrates aufgrund des Rats eines Novatianers das Verfahren vorgegeben, indem er die Teilnehmer auf-

44 Socr. H. E. 5, 10, 6-28; vgl. R. Lim, Public Disputation, Power and Social Order in Late Antiquity, Berkeley/Los Angeles/London 1995, 202f;; M. Wallraff, Der Kirchenhistoriker Sokrates. Untersuchungen zu Geschichtsdarstellung, Methode und Person (Forschungen zur Kirchen- und Dogmengeschichte 68), Göttingen 1997, 275ff., der gegenüber den Einzelheiten des Berichtes skeptisch ist; Leppin, Theodosius (wie Anm. 17), $83 \mathrm{f}$. 
forderte, auf der Grundlage der Väter eine Debatte zu führen, wobei er offenbar anwesend blieb. Nachdem auf diese Weise eine zielführende Debatte nicht zustande gekommen war, hatte er von allen teilnehmenden Gruppen Glaubensbekenntnisse eingefordert, sich zurückgezogen und war die Texte unter Gebet durchgegangen. Schließlich verlieh Gott ihm die Etkenntnis, daß das Nizänische Bekenntnis das richtige sei.

Justinian war da weitaus zurückhaltender. Weder griff er so massiv in das Verfahren ein, noch hatte er sich Glaubensbekenntnisse vorlegen lassen, geschweige denn, daß er kraft eigenen Glaubens darüber entschieden hätte; er beschränkte sich vielmehr auf einige Kompromißvorschläge. Auch wenn Justinian die persönliche Nähe Gottes gesucht hatte ${ }^{45}$ - wie sie ja jedem Christen offensteht -, so hatte er lediglich für die Einigung unter den $\mathrm{Ge}$ sprächsteilnehmern gebetet. Falls Justinian von dem Religionsgespräch unter Leitung des Theodosius wußte, muß es ihm zu denken gegeben haben, daß das Projekt 150 Jahre zuvor gelungen war, so daß seither die Gegner von Nizäa im theodosianischen Reich keine Gefahr mehr darstellten - während sein Religionsgespräch in einen Fehlschlag mündete.

Das Scheitern des Religionsgesprächs von Konstantinopel 532 führte zu keinen rabiaten Aktivitäten Justinians, doch erließ er 533 an die Bewohner Konstantinopels und einer Reihe anderer Städte, nicht aber Alexandreias, eine Konstitution, in der er sich unter Berufung auf die Lehre der Kirche und ihrer Priester eindeutig gegen Nestorius und Eutyches wie auch Apollinaris wandte, bei der positiven Formulierung des Glaubens jedoch weiterhin auf alle Formeln verzichtete, welche die Miaphysiten provozieren konnten, während er die theopaschistische Formel bestätigte. ${ }^{46}$ Allerdings verwies er in zeitgleichen Schreiben an den Patriarchen von Konstantinopel Euphemios und an Papst Johannes II. von Rom auch einmal auf die vier Konzilien, womit die Miaphysiten nicht einverstanden sein konnten. ${ }^{47}$ Eine Abweichung vom bisherigen Kurs bedeutet das indes nicht, weil ja schon auf dem Religionsgespräch des Jahres 532 Justinian die inhaltlich entleerte Akzeptierung von Chalkedon angeboten hatte. Beide Schreiben wurden übrigens in den

45 Wie sehr Justinian Respekt vor den Prärogativen kirchlicher Institutionen in dogmatischen Dingen zeigt, betont W. Enßlin, Justinian I. und die Patriarchate Rom und Konstantinopel, SO 35, 1959, 113-127.

46 CJ 1, 1, 6; vgl. Grillmeier, Jesus der Christus (wie Anm. 14), 362f.; Speigl, OS 44, 1995 (wie Anm. 14), 116f. bezieht die tuvós des $\$ 1$ auf die Akoimeten, die die Hauptzielscheibe der Kritik gewesen seien - möglicherweise zu Recht. Gray, Legacy (wie Anm. 11), 231 will den Einfluß des Leontius erkennen.

47 CJ 1, 1, 7; vgl. Speigl, OS 44, 1995 (wie Anm. 14), 118 ff.; s. auch Coll. Av. 84, 17. 
„Codex Iustinianus“ aufgenommen, so daß man nicht unterstellen darf, der Kaiser habe ein doppeltes Spiel getrieben.

Von einer schweren, systematischen Bedrängung der Miaphysiten kann auch in diesen Jahren keinesfalls die Rede sein; der Kaiser wurde zwar aktiver, aber er schlug die Tür nicht vor ihnen zu. ${ }^{48}$ Weitaus härter griff er die Akoimeten an, Mönche, die eine besonders radikale, den Theopaschismus verwerfende Deutung des Chalkedonismus vertraten und die versucht hatten, den Papst gegen Justinian in Stellung zu bringen. ${ }^{49}$

$\mathrm{Zu}$ einem Ausgleich gelangte Justinian mit dem wichtigsten Exponenten der chalkedonischen Gegenseite, Papst Johannes II., denn dieser konnte sich wenigstens zu einer indirekten Anerkennung der theopaschistischen Formel in einer leicht umakzentuierten Gestalt und einer Abkehr von den Akoimeten verstehen. ${ }^{50}$ In den Kirchen Konstantinopels sollte ferner künftig auf kaiserliche Anordnung ein Hymnos gesungen werden, der eine theopaschistische Wendung enthielt und der auch den Severianern genehm war. ${ }^{51}$ Damit hatte das chalkedonische Lager eine größere Geschlossenheit gewonnen, ohne sich zu deutlich gegenüber den Miaphysiten abzugrenzen.

Etwas anderes trat alsbald in den Vordergrund. Im November 533 war es bei einem Erdbeben in Konstantinopel zu einem Massenauflauf von protestierenden Gegnern des Chalcedonense gekommen, ${ }^{52}$ was man als Indiz ihrer Stärke nehmen konnte. Vor allem aber wartete man darauf, daß Severus nach Konstantinopel komme. Es bestand die Chance, daß der Kaiser selbst mit dem wichtigsten Miaphysiten spreche, wie er es ja ausdrücklich wünschte. Als der abgesetzte Bischof von Antiocheia 535 endlich eintraf, ${ }^{53}$ wurde er vom Kaiser mit allen Ehren empfangen und scheint ihn nachhaltig beeindruckt zu haben. Schon dies muß die Chalkedonier irritiert haben. Überdies gelangte

48 Hierin folge ich Uthemann, Augustinianum 39, 1999 (wie Anm. 11), 34ff.

49 Speigl, OS 44, 1995 (wie Anm. 14), 117; vgl. Mazal, Justinian (wie Anm. 9), 206.

50 Grillmeier, Jesus der Christus (wie Anm. 14), 357; Speigl, OS 44, 1995 (wie Anm. 14), $122 \mathrm{ff}$; Uthemann, Augustinianum 39, 1999 (wie Anm. 11), 34ff. Der Bedeutungsverlust der Akoimeten beschleunigte sich, s. Uthemann a.a.O., 35. Zur Umakzentuierung vgl. L. Abramowski, Die Mosaiken von S. Vitale und S. Apollinare in Classe und die Kirchenpolitik Kaiser Justinians, ZAC 5, 2001, 289-341, hier: $312 \mathrm{f}$.

51 Theoph. a m. 6028; vgl. Uthemann, Augustinianum 39, 1999 (wie Anm. 11), $38 f$.

52 Chron. Pasch. 629 B/C Dindorf.

53 Zur Datierung Stein, Histoire II (wie Anm. 9), 382 Anm. 1; vgl. Joh. Eph. Beat. Or. 48 (PO 18, 687). Ps.-Zach. Rhet. 9, 19 und Evagr. H. E. 4, 10 führen den Empfang des Severus auf den Einfluß Theodoras zurück, doch das ist nicht nötig, wenn man das Verhalten Justinians beim Religionsgespräch 532 vor Augen hat, wo er ja schon nach Severus verlangt hatte. 
535 mit Theodosios ein Freund des Severus dank massiver Unterstützung vom Hof auf den Bischofstuhl von Alexandreia - allerdings war sein Konkurrent ebenfalls ein Miaphysit aus der Richtung der Julianisten; ein Chalkedonier spielte bei den Auseinandersetzungen keine Rolle. Vor allem aber bestieg, ebenfalls 535, Anthimos den Bischofsstuhl von Konstantinopel, der sich als unsicherer Kantonist erwies. Zuvor hatte er als Bischof von Trapezunt an dem Religionsgespräch von 532 auf der Seite der Chalkedonier teilgenommen, jetzt erweckte er jedoch den Eindruck, er sei ein Severianer. Die Waage schien sich zu Ungunsten der Chalkedonier zu neigen.

Da trat Anfang 536 unversehens ein dritter kirchlicher Akteur auf den Plan, Agapet, der neue Bischof von Rom, ein entschiedener Anhänger Chalkedons, der vom ostgotischen König Theodahat in diplomatischer Mission in den Osten geschickt worden war. Er sollte - die Vorbereitungen zum Gotenkrieg waren im Gange - darum bitten, daß die kaiserlichen Truppen, die Italien bedrohten, abgezogen würden. Mit diesem Anliegen, das er möglicherweise auch gar nicht offensiv vertreten hat, drang er nicht durch; mit einem anderen erzielte er dafür einen um so größeren Erfolg: 54

Agapet, der schon zuvor wegen kirchenorganisatorischer Fragen mit Justinian in Streit geraten war, war von Mönchen, zu denen viele strenge Chalkedonier zählten, über die kirchenpolitischen Zustände in der Hauptstadt unterrichtet worden. Da gab es ja nach wie vor Zoras mit seiner Anhängerschaft, ${ }^{55}$ und da gab es eben einen Bischof, dessen Bekenntnis zu Chalkedon nicht eindeutig schien. Agapet trug dies dem Kaiser vor, der daraufhin die Absetzung des Konstantinopolitaner Patriarchen verfügte; dies wurde, durchaus nachvollziehbar, damit begründet, daß Anthimos durch die Translation von einem Bischofssitz auf den anderen und somit in unkanonischer Weise zum Amt bestellt worden war. ${ }^{56}$ Eine dogmatische Frage verknüpfte sich mit einer kirchenrechtlichen, und Agapet scheint dies genutzt zu haben.

Agapet inszenierte sich bei dieser Gelegenheit, wenn man dem „Liber pontificalis", der in dieser Passage eine zeitnahe, allerdings westliche, von

54 Zu Agapets Wirken in Konstantinopel E. Caspar, Geschichte des Papsttums. Von den Anfängen bis zur Höhe der Weltherrschaft, Bd. 2: Das Papsttum unter byzantinischer Herrschaft, Tübingen 1933, $221 \mathrm{ff}$.

$55 \mathrm{Zu}$ den Angriffen Agapets auf Zoras, vgl. Joh. Eph. Beat. Or. 2 (PO 17, 26ff.). Schließlich verwünschte Zoras ihn zu Tode.

56 Nov. Iust. 42. Joh. Eph. Beat. Or. 48 (PO 18, 685f.); vgl. Joh. Eph. H. E. 1, 42, wo er sicherlich zu Unrecht suggeriert, Anthimos habe von sich aus auf den Thron verzichtet. 
Sympathie für Agapet erfüllte Quelle repräsentiert, ${ }^{57}$ trauen darf, in ähnlicher Weise, wie wir es bei Zoras gesehen hatten: Er zeigte Parrhesie und beschimpfte den Kaiser nach Kräften, ja, er ging so weit, daß er diesen als neuen Diokletian bezeichnete.

Ein schlechter Kaiser hätte angesichts solcher Anwürfe mit Zorn reagiert, aber Justinian tat dies genauso wenig wie im Falle des Zoras. Bemerkenswert ist, was der „Liber pontificalis“ über dieses Ereignis schreibt: Tunc piissimus Augustus gaudio repletus bumiliavit se sedi apostolice et prostravit ante papam Agapetum. „Da demütigte sich der tieffromme Kaiser, von Freude erfüllt, vor dem apostolischen Sitz und warf sich vor dem Papst Agapet nieder." Was hier geschildert oder wenigstens imaginiert wird, ist eine Facette des Justinian-Bildes, die man nicht unbeachtet lassen sollte: Man traute dem Kaiser zu, sich ähnlich zu erniedrigen, wie es Theodosius vor Ambrosius getan hatte ${ }^{58}$ - daß nicht jeder es ihm zutraute, zeigt sich darin, daß die Handschriften auch die vagere Lesart adoravit statt prostravit anbieten; das bumiliavit aber ist textkritisch gesichert. Der Kaiser hätte somit, ganz gleich, wie man die Lesung auffaßt, seinen Richtungswechsel in Konstantinopel damit motiviert, daß er von einem geeigneten Mann, eben Agapet, belehrt worden wäre. Das war nach christlicher Auffassung untadelig. Allerdings geht aus späteren Bemerkungen Justinians hervor, daß er durchaus noch Anthimos zur Umkehr zu bewegen versucht hatte, ${ }^{59}$ daß die Entscheidung also keineswegs schlagartig erfolgte. ${ }^{60}$ Welches Ansehen Agapet dennoch genoß, zeigen Glaubensschriften Justinians und des Patriarchen Menas, des Nachfolgers von Anthimos, die Agapet vorgelegt wurden, der im übrigen in seiner zustimmenden, auch die Zugeständnisse des

57 Zur Agapet-Vita im „Liber Pontificalis“ s. L. Duchesne, Introduction, in: ders., Le liber pontificalis. Texte, introduction et commentaire Bd. 1 (BEFAR 3, 1), Paris 1886, ICCLXII, hier: XLVIII, der zeigt, daß sie von einem Zeitgenossen verfaßt sein muß; zum Autor auch P. Hildebrand, Die Absetzung des Papstes Silverius (537). Eine quellenkritische Untersuchung, HJb 42, 1922, 213-249, hier: 217, der ihn auf kurz nach 538 datiert.

58 Bei der Begrüßung des Sabas durch Justinian taucht schon früher in einem vergleichba-

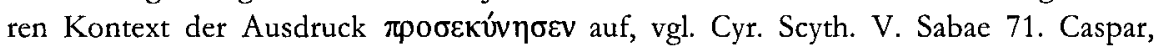
Papsttum II (wie Anm. 54), 225, der den Bericht des „Liber pontificalis“ anzweifelt, sieht hier lediglich eine kurzfristige Unterbrechung von Justinians in seinen Augen cäsaropapistischem Vorgehen.

59 Nov. Iust. 42 pr.

60 Agapet berichtet selbst in einem Brief an den Bischof Petros von Jerusalem von diesem Ereignis (ACO III, 152f.) - wobei er sich auf die Unterstützung der $\beta \alpha \sigma \lambda \varepsilon \varepsilon \hat{\varsigma} \varsigma$ - also offenbar auch Theodoras beruft (153, Z. 1f.; vgl. 10 zur Einsetzung des Menas) 
Johannes im wesentlichen bestätigenden Antwort kühl hervorhob, daß einem Laien keine Lehrautorität zustehe. ${ }^{61}$

Ein solcher Triumph, wie ihn Agapet erzielt hatte, mußte unter Chalkedoniern als Ausdruck überlegener Heiligkeit gewertet werden. Ps. Zacharias Rhetor indes, der Miaphysit, schreibt den Erfolg der Tatsache zu, daß Agapet wie Justinian lateinischsprachig gewesen sei, außerdem habe Justinian ihn aufgrund der Bedeutung Italiens begünstigt. ${ }^{62}$ Das ist angesichts der kriegerischen Entwicklungen sicherlich nicht von der Hand $z u$ weisen, wäre aber doch zu kurz gegriffen, zumal Justinian auch in kritischen Situationen seiner Italienpolitik keine Scheu hatte, hart gegen Päpste vorzugehen. ${ }^{63}$ Man sollte eine personalisierende Interpretation nicht von vornherein ausschließen: Offenbar war Justinian von der Gestalt des Papstes nachhaltig beeindruckt andererseits hatte Anthimos stillschweigende Übereinkünfte mit Severus und Theodosios I., dem Patriarchen von Alexandreia, getroffen, ${ }^{64}$ so daß Justinian allen Grund hatte, diesem Personenkreis zu mißtrauen.

Alles schien nach dieser Begegnung auf eine ideale Beziehung zwischen Kaiser und Papst hinauszulaufen, da geschah etwas Unvorhersehbares: Agapet starb am 22. April 536. Hier hatten die Zeitgenossen gewissermaßen die Nagelprobe für das Wirken des Heiligen Mannes vor sich. Der rasche Tod eines Beteiligten in einer entscheidenden Phase stellte die Wahrhaftigkeit seiner Lehren evident in Frage. Die Gefolgsleute des Zoras wußten zu berichten, daß ihr Held Agapet für seine lasterhaften Äußerungen eine mehrere Meter lange Zunge in den Hals gebetet habe, an der er erstickt sei. ${ }^{65}$ Rasches Handeln war angesichts solcher Triumphgefühle gefordert.

Die Anhänger Agapets ließen sich in der Tat nicht lähmen, sie drangen auf ein Konzil der in Konstantinopel präsenten Bischöfe, auf eine Endemousa. Die vereinigten Mönche Konstantinopels sowie Vertreter vor allem palästinischer Klöster, die einen Stützpfeiler des Chalkedonismus bildeten und von deren Brüdern eine nicht geringe Zahl in der Hauptstadt lebte, ${ }^{66}$ setzten sich

61 Coll. Av. 89-91, insbes. 91, Z. 3 mit Speigl, OS 44, 1995 (wie Anm. 14), 130 ff.

62 Ps.-Zach. Rhet. 9, 19.

63 Vgl. Noethlichs, ZAC 4, 2000 (wie Anm. 26), $126 f$.

64 Vgl. den Brief des Severus an Theodosius bei Ps.-Zach. Rhet. 9, 23.

65 Welche Triumphgefühle der Tod Agapets bei seinen Gegnern auslöste, zeigt der Bericht bei Joh. Eph. Beat. Or. 2 (PO 17, 30f.) - Ich sehe kein Indiz dafür, daß Justinian sich in dieser Situation als Testamentsvollstrecker Agapets gefühlt habe (so Schwartz, Kirchenpolitik Justinians [wie Anm. 10], 287).

$66 \mathrm{Zu}$ ihnen K. Trampedach, Reichsmönchtum? Kyrill von Skythopolis und das politische Selbstverständnis der Mönche Palästinas im 6. Jahrhundert, Millennium 2, 2005, 271-295. 
an die Spitze dieser Bewegung. Wieder sind wir über die Versammlung recht gut unterrichtet, da Protokolle und Dokumente des Konzils vorliegen. ${ }^{67}$ Der Kaiser hatte es einberufen, der noch von Agapet geweihte Nachfolger des Anthimos, Menas, stand ihm als Ortsbischof vor. Eröffnet wurde es bereits am 2. Mai 536, wenige Wochen nach Agapets Tod.

Man bemühte sich um formale Korrektheit: Die Mönche fragten eigens, ob sie eintreten dürften, ebenso der Vertreter der Politik, der Referendar Theodoros ( $(57)$, der erläuterte, daß der Kaiser eben auf Bitten der Mönche das Konzil einberufen habe, und das entsprechende Schreiben verlesen ließ: 68

Der Brief berichtet nach langen Bekundungen der Loyalität, wie $\mathrm{zu}$ erwarten, daß Anthimos zu Unrecht Bischof geworden sei und Unfrieden gestiftet habe. Dann folgt eine bemerkenswerte Beschreibung der kaiserlichen Rolle: Er hätte natürlich die Häretiker vertreiben können, erklären die Mönche, doch habe er es vorgezogen, den kanonisch korrekten Weg zu gehen und den Priestern zu gehorchen, zumal dem Urteil des Bischofs von Rom. Da habe Gott Agapet nach Konstantinopel entsandt. ${ }^{69}$ Dieser habe, von den Mönchen über die schlimmen Zustände informiert, das Gespräch mit Anthimos verweigert. Überdies hätten andere Bischöfe und Mönche den Kaiser um die Bekehrung der Häretiker oder ihre Vertreibung aus Kirche und Stadt ersucht (Z. 21 ff.). Diesen ganz berechtigten Bitten sei Agapet zuvorgekommen, indem er die Häretiker abgesetzt habe und die Bitte der Mönche an den Kaiser, das zu Ende zu führen, was die Mönche erbeten hätten, weitergeleitet habe (Z. 36ff.).

Nach der Darstellung des Briefes der Mönche, der, wie gesagt, an den Kaiser gerichtet und von ihm weitergeleitet worden war und daher gewiß nicht das Ziel haben konnte, den Kaiser als Schwächling dastehen zu lassen, ist eindeutig Agapet der Motor der Geschehnisse. Agapet ergreift die Initiative, der Kaiser folgt ihm. Das Handeln des Papstes zwang demnach den Kaiser zu einer Reaktion. Offenbar erwartete man, daß Justinian diese Deutung teile, und das erscheint auch nach dem, was wir gesehen haben, durchaus plausibel: Der Kaiser hatte bislang stets seinen Respekt vor religiösen Instanzen demonstriert, er hatte sie um ihren Rat ersucht - was innerhalb seiner bisherigen Politik konsistent war.

67 ACO III, 27-189.

68 Zu ihm PLRE IIIB, 1248.

69 ACO III, 132, Z. 4 ff. 
Im zweiten Teil des Briefes wird kurz der Tod Agapets erwähnt (S. 133, 1-3); der Kaiser solle jetzt, da Agapet Gott näher sei, seine Maßnahmen zur Vollendung führen, dann werde Gott ihm alle Feinde unterwerfen und ihn im Himmel vielfach belohnen.

Man könnte diese Konzilsdokumente als Belege für die politische Schwäche des Kaisers lesen, doch sind sie offenbar Spiegel kaiserlichen Wollens. Er war demnach, wie sich erneut zeigt, nicht bestrebt, als derjenige zu erscheinen, der sich in kirchlichen Belangen kraft eigener Gewalt durchzusetzen vermochte. Dementsprechend war die Kirchenversammlung umgekehrt nicht zuletzt eine Inszenierung von Loyalität gegenüber dem Kaiser, dessen Rechtgläubigkeit und guter Wille allenthalben hervorgehoben wurde.

Allerdings blieb als eine Peinlichkeit bestehen, daß es nicht gelang, des abgesetzten Anthimos habhaft zu werden, der den Vorladungen keine Folge geleistet hatte, ${ }^{70}$ daß also in diesem Punkte die Grenzen der Macht des Kaisers in der Hauptstadt sichtbar wurden. Die Beschlüsse als solche entsprachen den Vorstellungen Agapets und nunmehr wohl auch des Kaisers. Anthimos, Severus und andere Miaphysiten wurden als häretisch gebrandmarkt. Der Kaiser sanktionierte diese Beschlüsse am 25. Juli 536 mit der Novelle 42, wobei er im Proömium ausdrücklich erklärt, daß er sich dem Votum der Priester anschließe; ${ }^{71}$ mit dieser Novelle bekannte der Kaiser sich überdies ausdrücklich zu Chalkedon und verbannte eine Reihe von Miaphysiten darunter übrigens auch, namentlich genannt, Zoras ${ }^{72}$ - aus Konstantinopel und überhaupt den bedeutenderen Städten. Auch in den nächsten Jahren setzte er sie mit Härte und relativ großer Konsequenz durch. Die Miaphysiten wurden aus der Öffentlichkeit gedrängt. ${ }^{73}$

Es wäre unangemessen, hier mit Eduard Schwartz von einem Triumph des Kaisers zu sprechen. ${ }^{74}$ Er hatte zwar jetzt für sich eine Lösung gefunden, doch der integrative Ansatz, den er fast zehn Jahre lang erprobt hatte, war gescheitert. Es war kein langgehegter Plan des Kaisers, ein bestimmtes Dog-

70 Laut Joh. Eph. Beat. Or. 48 (PO 18, 686) hatte Theodora ihn verborgen; allerdings ist der Bericht über Anthimos von zahlreichen Fehlern durchsetzt.

71 In Nov. Iust. 42, 3, pr. erklärt er in Hinblick auf Zoras, daß das Votum der Priester allein ausreiche und durch das des Kaisers lediglich verstärkt werde.

72 Nov. Iust. 42.

73 Bemerkenswert die Bewertung durch Liberatus Brev. 159 (ACO II, 5, 138, Z. 32f.): Hoc ergo modo unitas facta est ecclesiarum anno $X$ imperii gloriosi Iustiniani Augusti. Bei den chalkedonischen Mönchen Palästinas wurde diese Synode in eine Reihe mit den vier Ökumenischen gestellt, s. Schwartz, Praefatio zu ACO III, S. X; vgl. auch das Lob bei Evagr. H. E. $4,11$.

74 Schwartz, Kirchenpolitik Justinians (wie Anm. 10), 290. 
ma zu etablieren, der hier durchgesetzt wurde. Offenbar hatte er vielmehr nach seinen langen Bemühungen den Eindruck gewonnen, daß letztlich eine klare Positionsbestimmung die Grundlage des Erfolges sei und mochte sich durch das Charisma Agapets einerseits, die Tricksereien von Anthimos und seinen Freunden andererseits bestätigt fühlen. Diese Politik exekutierte er fortan mit zunehmender Härte.

Es hat sich gezeigt, daß die ersten neun Jahre der justinianischen Religionspolitik im Zeichen der Kontinuität stehen. Von Anfang an zielte der Kaiser darauf, die Miaphysiten einzubinden und damit auf ihr Erstarken zu reagieren, nicht so sehr darauf, das eigene Bekenntnis in aller Schärfe zu verbreiten, zumal dies in den Provinzen ohnehin kaum möglich war. Der aggressive Tonfall der antihäretischen Gesetze täuscht, denn er richtet sich gar nicht gegen die Miaphysiten, sondern gegen gemeinsame theologische Gegner. Sie gehören in den Kontext anderer Gesetze, die gegen Gruppen wie die Heiden oder Samaritaner gerichtet sind. Die Identifizierung gemeinsamer Feinde sollte wohl eine gemeinsame Identität begründen.

Kontinuität bestand auch darin, mit welchem Habitus der Kaiser seine Religionspolitik praktizierte. Zwar spürte er offenbar, und das entsprach nun einmal der kaiserlichen Rolle, die Verpflichtung, sich für den wahren Glauben einzusetzen, zwar hatte er ohne Zweifel auch bestimmte Vorstellungen davon, wie der wahre Glaube aussehe - das Konzil von Chalkedon war für ihn wenigstens formal nicht antastbar, die theopaschistische Formel vertrat er mit Konsequenz -, doch besaß er eine prinzipielle, zumindest zur Schau getragene Bereitschaft, sich belehren zu lassen. Hierin wird eine gewisse Widersprüchlichkeit in der Rolle des christlichen Kaisers sichtbar: Er mußte den wahren Glauben vertreten, aber er war, jedenfalls für ernsthafte Theologen, nicht derjenige, der den wahren Glauben von sich aus zu erkennen vermochte.

Die Rolle dessen, der sich belehren läßt, nahm Justinian so ernst, daß er bei religiösen Konflikten stets als der zuhörende, zur Buße bereite und nicht zornmütige Herrscher auftrat; ${ }^{75}$ selbst im Falle des Zoras, der die Miaphysiten in provozierender Weise vertrat und dessen Begegnung mit dem Herrscher selbst durch eine feindselige Quelle in dieser Weise geschildert wird.

75 Selbst gegenüber den Manichäern gab es Gesprächsversuche, s. Lim, Public Disputation (wie Anm. 44), 105f. Noethlichs, ZAC 4, 2000 (wie Anm. 26), 126 betrachtet die ganze Regierungszeit Justinians als die eines ,wirklich Suchenden". 
Justinians Milde ging so weit, daß sie auf der Synode von 536 sogar sanft getadelt wurde. ${ }^{76}$

Was sich wandelte, waren die Methoden. Schien es Justinian zunächst ausreichend, die gemeinsamen Feinde zu identifizieren, so zeigt das Religionsgespräch von 532, daß er nunmehr zu inhaltlichen Aussagen gelangen wollte. Doch all das scheiterte; es befriedete die Verhältnisse nicht. Erst die Begegnung mit Agapet und der Betrug der Miaphysiten scheint einen Bruch bewirkt zu haben, und es mußte der Eindruck entstehen, daß die Endemousa 536 schließlich zu einem klaren Ergebnis geführt hatte. Wurden nicht Unruhestifter wie Zoras vertrieben? Hatte der Kaiser sich nicht klar auf die Seite der Chalkedonier gestellt? Hatte der Kaiser nicht zu einem klaren Bekenntnis gefunden?

Tatsächlich wurde die Synode von 536 zeitweise in eine Reihe mit den vier großen, Ökumenischen Konzilien gestellt, tatsächlich agierte Justinian nun ungleich selbstbewußter als zuvor: Das Mönchtum wurde diszipliniert, Papst Vigilius merklich härter angefaßt als seine Vorgänger, in Alexandreia ein konsequent chalkedonischer Kurs gesteuert. War damit die Religionspolitik Justinians zu einem Zielpunkt gelangt?

Für einige Jahre mochte es so scheinen, doch der Eindruck verflüchtigte sich bald, nicht nur weil der Versuch, in Alexandreia Ruhe zu schaffen, scheiterte. Seit den vierziger Jahren griff Justinian verstärkt mit theologischen $\mathrm{Ar}$ gumenten in die Kirchenpolitik ein. ${ }^{77}$ Einen ersten Höhepunkt erreicht dies im origenistischen Streit. ${ }^{78}$ In dem 542/3 publizierten Edikt gegen die Origenisten forderte er etwa, nachdem er sich eingehend zu theologischen Probleme geäußert hat, die Einberufung einer Endemousa, um diese Fragen entsprechend zu behandeln; ${ }^{79}$ anders als in der Novelle 42, mit der er auf das Konzil von 536 reagierte, erklärte er hier nicht, daß er sich dem Votum der kirchlichen Autoritäten anschließe. Vielmehr nahm er die Lehrautorität eines Laien

76 ACO III, 43, Z. 19-24.

77 Vgl. Meier, Zeitalter Justinians (wie Anm. 10), $278 f$.

78 Der Streit, der von gebildeteren Mönchen ausging (vgl. E. Schwartz, Kyrillos [wie Anm. 10], 388 sowie D. Hombergen, The Second Origenist Controversy. A New Perspective on Cyril of Scythopolis' Monastic Biographies as Historical Sources for Sixth-Century Origenism, Rom 2001, 231ff., der allerdings hervorhebt, daß auch auf der Gegenseite Gebildete standen) brachte eine Intellektualisierung der Debatten mit sich, die möglicherweise Justinians Vertrauen in seine eigenen geistigen Gaben stärkte.

79 ACO III, 189-214, insbes. 207, Z. 30-208, Z. 17. Natürlich waren auch an diesen Maßnahmen Bischöfe nicht unbeteiligt. Justinian handelte, so heißt es, auf Initiative Ephraims von Antiocheia, setzte dann seine Schrift auf und überließ sie Menas und seiner Synode zur Zustimmung, s. Cyr. Scyth. V. Sab. 191. 
in Anspruch, die der verehrte Agapet ausdrücklich abgelehnt hatte. ${ }^{80}$ Es war nicht mehr eine kurze Kompromißformel wie die theopaschistische, um die es hier ging, sondern eine ausführliche theologische Erörterung.

Von einem völligen Wandel der Politik Justinians ist indes auch für die spätere Epoche nicht auszugehen. Das Beharren auf der theopaschistischen Formel in den Anfängen Justinians zeigt, auch wenn die Wendungen Kompromißcharakter hatten, daß der Kaiser an bestimmten Überzeugungen festhielt; umgekehrt lassen sich auch in der späteren Zeit immer wieder Versuche des Kaisers beobachten, mit Vertretern anderer Richtungen ins Gespräch zu kommen; immer wieder ließ er sich offenkundig auch von einzelnen Christen beeindrucken, selbst wenn er ihren Lehren ablehnend gegenüberstand. ${ }^{81}$ Doch eine religiöse Integration führte er nicht herbei; vielmehr bildete sich gerade unter seiner Herrschaft eine eigene miaphysitische Kirche heraus. Noch seine Hinwendung zum Aphthartodoketismus am Ende seines Lebens mag ein Versuch gewesen sein, Chalkedonier und Miaphysiten aneinander anzunähern. Eben dadurch aber isolierte der Kaiser sich vollends, der nunmehr auch bei den Chalkedoniern als Häretiker galt.

80 Coll. Av. 91, 3; vgl. Meiers Deutung der Religionspolitik in dieser Phase: Zeitalter Justinians (wie Anm. 10), 273ff.

81 Seine Bereitschaft, auf Heilige Männer zu hören, bleibt auch später bestehen, selbst im Falle von Miaphysiten, s. Joh. Eph. Beat. Or. 47 (PO 18, 680); die Provokationen des Mares, die nach 536 erfolgt sein dürften, nahm er gelassen hin, s. Joh. Eph. Beat. Or. 36 (PO 18, 428ff.). 